\title{
Modifications to the warm Spitzer data reduction pipeline
}

\author{
Patrick J. Lowrance ${ }^{a}$, Sean J. Carey ${ }^{a}$, Jessica E. Krick ${ }^{a}$, Jason A. Surace ${ }^{a}$, William J. Glaccum ${ }^{\mathrm{a}}$, Iffat \\ Khan ${ }^{\mathrm{a}}$, James G. Ingalls ${ }^{\mathrm{a}}$, Seppo Laine ${ }^{\mathrm{a}}$, Carl Grillmair ${ }^{\mathrm{a}}$ \\ ${ }^{a}$ Spitzer Science Center, 1200 East California Blvd, Pasadena, CA, USA 91125
}

\begin{abstract}
The Spitzer Space Telescope Infrared Array Camera (IRAC) basic calibrated data reduction pipeline is designed to take a single raw frame from a single IRAC detector and produce a flux-calibrated image that has had all well-understood instrumental signatures removed. We discuss several modifications to the pipeline developed in the last two years in response to the Spitzer warm mission. Due to the different instrument characteristics in the warm mission, we have significantly changed pipeline procedures for masking residual images and mitigating column pulldown. In addition, the muxbleed correction was turned off, because it is not present in the warm data. Parameters relevant to linearity correction, bad pixels, and the photometric calibration have been updated and are continually monitored.
\end{abstract}

Keywords: Infrared instrumentation, infrared detectors, astronomy, spacecraft instruments, Spitzer Space Telescope, IRAC

\section{INTRODUCTION}

The Spitzer Space Telescope ${ }^{1}$ is the fourth of NASA's great observatories and has made paradigm-shifting discoveries using its three infrared instruments, the InfraRed Array Camera ${ }^{2,3}$ (IRAC), the Infrared Spectrograph ${ }^{4}$ (IRS) and the Multi-band Imaging Photometer for Spitzer ${ }^{5}$ (MIPS), covering a wavelength range from 3.6 to $160 \mu \mathrm{m}$, during its $\sim 5.5$ year primary mission. The duration of the primary mission plus the three month in-orbit checkout and science validation (5.7 years of cryogenic lifetime) far exceeded the mission lifetime goal of five years. The success of the passive cooling of Spitzer by using an Earth-trailing orbit and judicious use of the makeup heater, which evaporated cryogen to actively cool the telescope mirror to permit observations with the longest wavelength (MIPS and IRS) instruments, were key elements in the great length of the cryogenic mission.

Prior to the end of the cryogenic mission, it was noted that the passive cooling would continue to achieve telescope and multiple instrument chamber (MIC) temperatures of $\sim 30 \mathrm{~K}$. Thermal models assuming $3 \mathrm{~mW}$ of power for operating IRAC predicted MIC temperatures of between 25 and $29 \mathrm{~K}$ and primary mirror temperatures of $24-25 \mathrm{~K}$. The thermal background of the telescope and the noise properties ${ }^{6}$ of the two InSb arrays of IRAC for passively cooled Spitzer provide comparable sensitivity to the cryogenic observations at 3.6 and $4.5 \mu \mathrm{m}$. A science case ${ }^{7}$ for an extended mission (called the warm mission in the remainder of this article) using the shortest wavelength cameras of IRAC was prepared and a four year (initial two year plus two year extension) post-cryogenic extended mission was awarded by NASA.

On May 15, 2009 the Spitzer Space Telescope exhausted the last of its cryogen. As the telescope and instruments slowly warmed to their new equilibrium temperature, numerous changes were made to IRAC operations ${ }^{8}$ and its data reduction pipeline in order to improve IRAC's performance at the new temperature. The applied bias on the arrays and the operating temperature were modified during the initial two months of science observations to improve the final sensitivities. The changes were implemented after it was determined that the power dissipated by the instrument was lower than predicted. The lower power dissipation allows the instrument to be run at a lower temperature than initially planned, with a significant improvement in noise properties and sensitivity. During the initial warm-up and settling, the arrays were tested at several biases and temperatures. From 18 September 2009 onward, the voltages have been set to their final values of $500 \mathrm{mV}$ of applied bias and array temperatures to $28.7 \mathrm{~K}$. Lower temperatures and higher applied biases produce an improved effective throughput and lower flux conversion values of the data.

\section{CALIBRATION CHANGES}

We distinguish the properties of warm IRAC data from those in cryogenic mission that necessitated changes in the data calibration pipeline. In general, the warm and cryogenic data are very similar and the optical properties such as image noise pixels are indistinguishable. The lack of changes in the optical properties is not unexpected as the linear expansion

Space Telescopes and Instrumentation 2012: Optical, Infrared, and Millimeter Wave, edited by Mark C. Clampin,

Giovanni G. Fazio, Howard A. MacEwen, Jacobus M. Oschmann, Jr., Proc. of SPIE Vol. 8442, 844238

(C) 2012 SPIE - CCC code: 0277-786/12/\$18 - doi: 10.1117/12.926944 
in materials in the optical path is expected to be less than one part in $10^{-5}$ at these temperatures. Changes in the applied calibrations such as bias (dark) subtraction, linearity solution and flat-field are compared. Note that while the calibrations are different and in some cases larger in magnitude for warm data, the data are still calibrated to the same level of accuracy and the images produced by the Spitzer Science Center pipeline are science quality.

\subsection{Bias}

Since IRAC does not use a photon shutter for a dark measurement, a pre-selected region of low zodiacal background in the north ecliptic cap is observed to create a "skydark." During each campaign, a library of skydarks of all frametimes is observed, reduced, and turned into skydarks with the pipeline. In addition to a stable bias pattern, the bias of the Fowler sampled images also depends on the interval from the last data collection. The effect is mainly a second order dependency, but the first order correction is sufficient for science purposes. For the cryogenic mission, this history dependent variation was measured pre-flight in the lab using shutter closed measurements. The character of this variation is very different in the warm mission and the cryogenic lab calibration no longer applies. An on-orbit warm calibration of this effect is in progress using skydark frame data taken with a wide variety of intervals. For both channels, the effect is $\sim 10 \times$ that of the cryogenic mission. As in the cryogenic effect, it appears to be repeatable; that is, the same magnitude and pattern of the bias are produced for observations with the same cadence. As the skydarks are taken in a similar exposure time, but every observation's cadence is different, there does exist a remaining bias level and pattern in every frame. As the effect is more significant in the warm mission, removal is a limiting factor for science observations that require high sensitivity for low surface brightness features, such as investigations of galactic halos and intracluster light. This effect is most significant for data taken without dithering, as the largest variation occurs for small time differences between frames.

For the warm mission calibration pipeline, skydark suites are taken, reduced, and used in the same manner as in the cryogenic mission. In addition to the higher bias level discussed above, clusters of bad pixels at 4.5 microns result in a series of artifacts (notably column pulldown, see section 3.2) being present in every frame. Since they are similar in
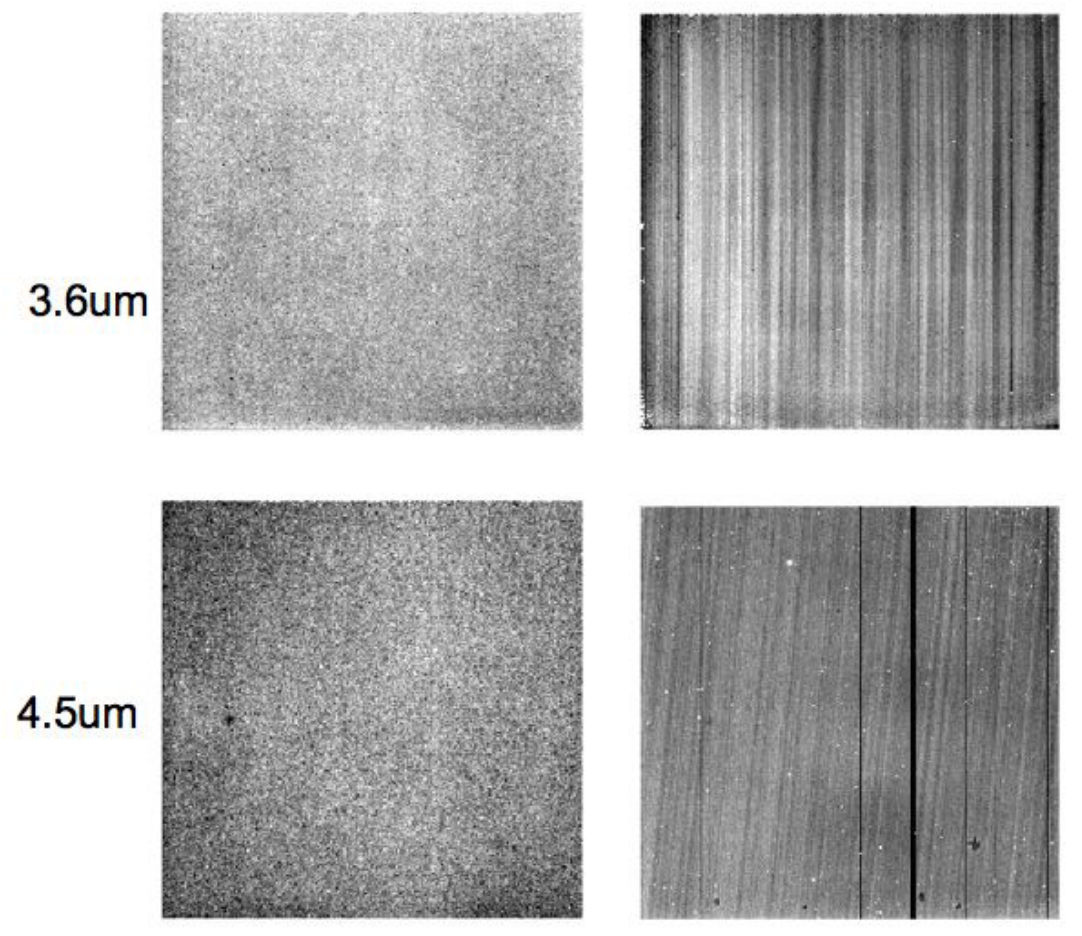

Figure 1. The bias as measured from a standard field assembled into a $12 \mathrm{~s}$ skydark ensemble created in the cryogenic mission (left) and during the warm mission characterization period (right). The column pulldown artifact is apparent in five columns of the 4.5 micron channel in the warm mission darks. Its strength is the same percentage of the background for all the frame times. The increased striping is a result of the more significant bias and not having a labdark subtracted. 
counts for frames of similar exposure times, these artifacts are mostly removed during the sky-subtraction stage in the pipeline. Median counts of the bias frames have increased significantly from $\sim 30 \mathrm{DN} / \mathrm{s}$ in cryogenic mission to $\sim 100$ $\mathrm{DN} / \mathrm{s}$ in warm mission in channel 1, and from $\sim 3 \mathrm{DN} / \mathrm{s}$ in cryogenic mission to $\sim 12 \mathrm{DN} / \mathrm{s}$ in warm mission in channel 2.

\subsection{Flat Field (Gain Map)}

The pixel-to-pixel gain variations (commonly called the flat-field) were re-measured during IRAC Warm Instrument Characterization (IWIC) and again every two weeks during the regular warm mission. These measurements were made in a similar manner as during the cryogenic mission; highly-dithered imaging of the brightest parts of the zodiacal cloud (fields in the Ecliptic plane) were differenced with the low background skydarks and then the per-pixel gain normalization of each array was determined. Flat-fields are taken with a similar cadence to the skydark observations to minimize the residual bias pattern. Overall, the flat-field has remained essentially unchanged relative to the cryogenic mission. There are, however, small gradients and other changes at the 1\%-3\% level, so the two are not interchangeable. Similarly, the normalization of the flats has changed slightly, so for self-consistency all data calibration is either cryogenic or warm, and not both. The flat-field measurements over the first two years of the warm mission were combined into a 'super-flat' that is accurate $(1 \sigma)$ to $0.3 \%$ at $3.6 \mu \mathrm{m}$ and $0.15 \%$ at $4.5 \mu \mathrm{m}$, and that calibration is now used for all science data.

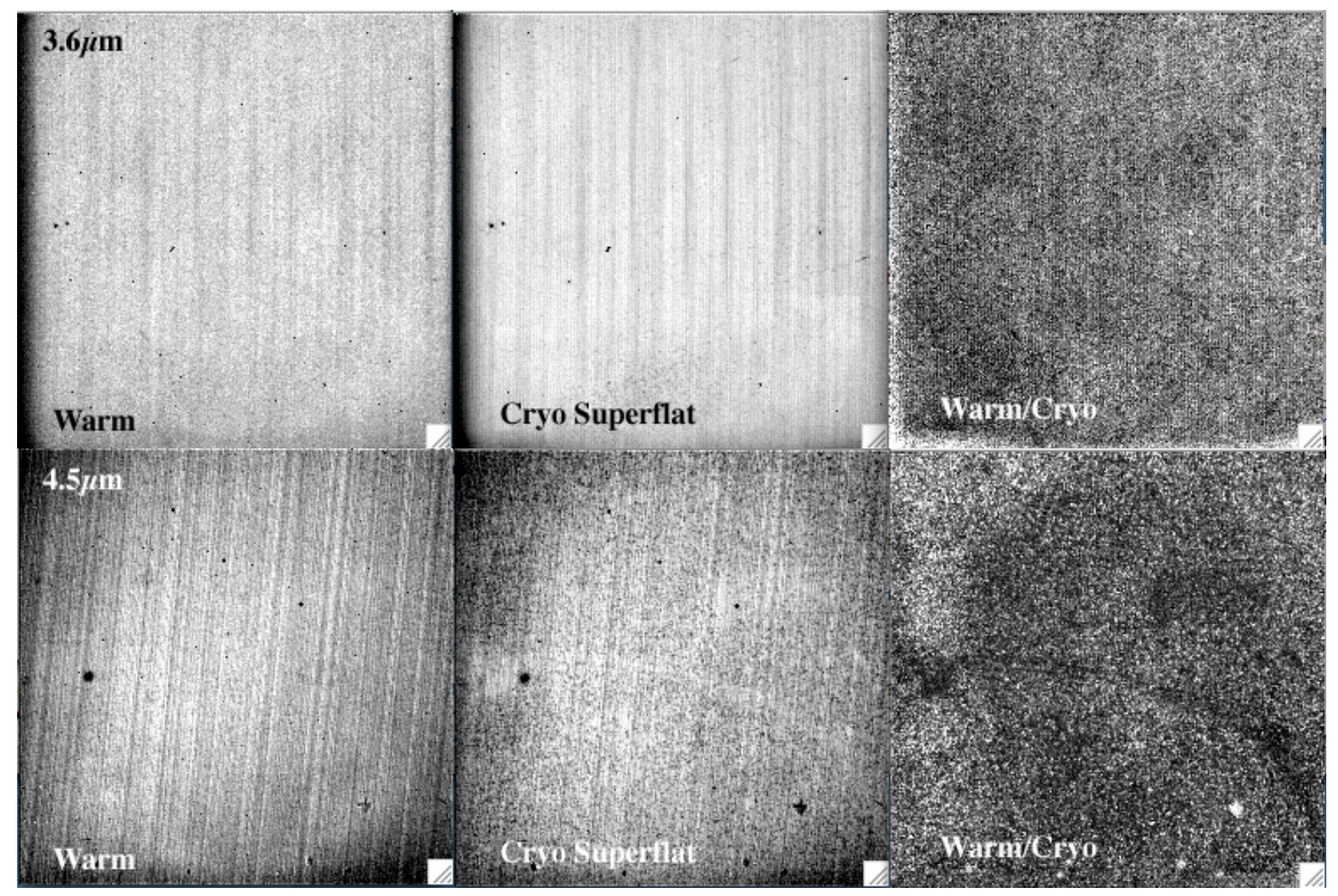

Figure 2: Warm (left), cryogenic (middle) and ratio of warm to cryogenic (right) flats at $3.6 \mu \mathrm{m}$ (top) and $4.5 \mu \mathrm{m}$ (bottom). Largescale variations at the level of a few percent are seen.

\subsection{Linearity}

The IRAC detectors have a non-linear response - the conversion from detected flux to data numbers is not a simple constant. In the cryogenic mission, this non-linearity was corrected based on ground calibration of the instrument using special test equipment, and was rederived and updated in-flight through numerous observations. During the beginning of warm data characterization, it was found that both the non-linearity and well-depth varied as functions of applied voltage on the arrays and the array temperature. Due to the changes, the linearity correction for each array is quite different than the cryogenic solution. Both arrays are significantly more non-linear in response when compared to the cryogenic 
mission. Unfortunately, due to the lack of uniform illuminators for the array, it is extraordinarily difficult to recalibrate the linearization in-flight with the accuracy of the ground-based cryogenic linearization.

The linearity calibration has recently been derived in flight from the photometry of sources observed by the SERVS exploration science program vs. the same sources observed by the SWIRE Legacy science program during cryogenic operations. This field was sampled at a variety of frametimes and with a range of sources of known brightness. A quadratic solution of is still applied for both channels, i.e., we model the detector response as

$$
\mathrm{DN}_{\mathrm{obs}}=\mathrm{DN}_{\text {true }}+\alpha \mathrm{DN}_{\text {true }}^{2}
$$

but the linearity coefficient, $\alpha$, has increased from $-1.9 \times 10^{-6}$ to $-3.5 \times 10^{-6}$ at $3.6 \mu \mathrm{m}$ and from $-2.7 \times 10^{-6}$ to $-4.1 \times 10^{-6}$ at $4.5 \mu \mathrm{m}$. As a result of the arrays being more non-linear, the effective well depth for each channel has been reduced by $\sim 33 \%$ from 45,000 DN to $\sim 30,000 \mathrm{DN}$. In terms of source brightness, warm IRAC saturates at 323 and $364 \mathrm{mJy} \times$ sec at 3.6 and $4.5 \mu \mathrm{m}$, respectively, for sources that are centered on an image pixel. Despite the more significant non-linearity, fluxes below saturation are correct to better than $1 \%$ just as in the cryogenic mission. A single solution is used for each array although nonlinearity varies slightly from pixel to pixel $^{9}$. The linearity solution for the observations before the final bias and temperature determination is less certain. Currently, the initial warm data characterization linearity solution is applied to the data taken while the temperature is floating. Since the array bias is the more important variable in determining linearity, this solution should be reasonable, but not strictly correct, as warm tests have shown that the linearity is also a function of temperature. Unfortunately, there is not enough data for sources of known brightness in the 12 August - 18 September 2009 time interval with which to construct an independent linearity solution. Judicious scheduling during this time period was used to observe primarily faint sources that are more robust to uncertainties in linearization. Users should examine the raw data numbers for sources that are suspected of being near saturation.
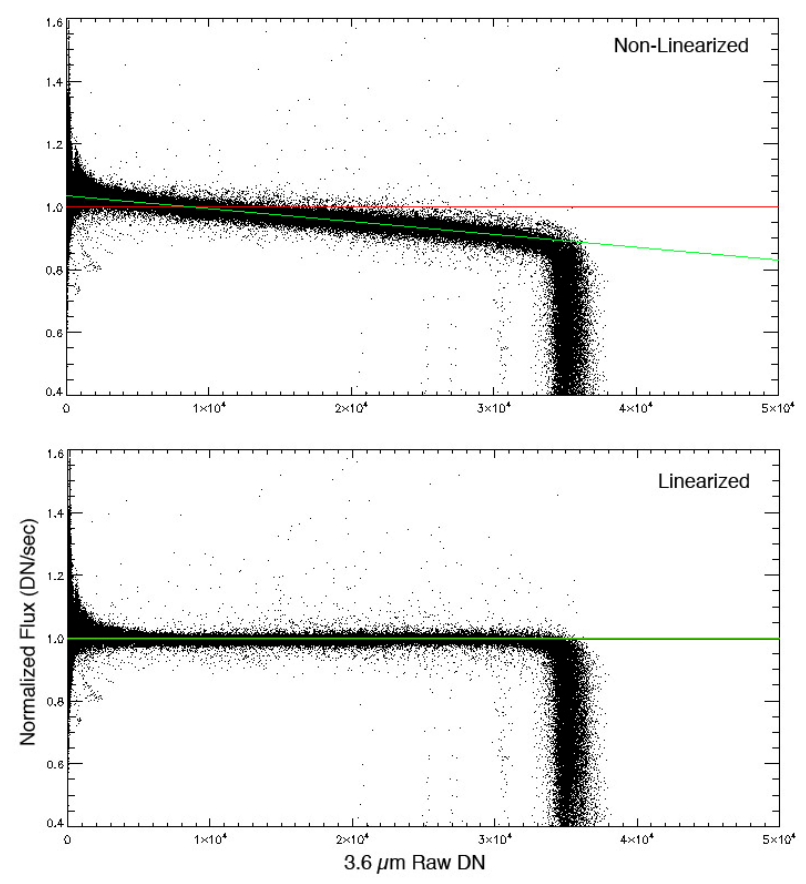

Figure 3. Warm linearity solution at $3.6 \mu \mathrm{m}$. The upper panel shows the raw DN compared to the true DN/s for each source in the SERVS field. The true DN/s are known from cryogenic observations of this field. The green line is the linearization solution and the bottom panel shows data with the linearity applied. At about 33,000 DN, the linearization starts to fail, and that sets the effective well depth. 


\section{DATA CALIBRATION PIPELINE}

The IRAC Level 1 (BCD; Basic Calibrated Data) pipeline is designed to take a single Level 0 ("raw") image from a single IRAC detector and produce a flux-calibrated image that has had all well-understood instrumental signatures removed. The IRAC pipeline consists of two principal parts: the data reduction software modules and the calibration server. The individual modules each correct a single instrumental signature. They are written as standalone code executable from the UNIX command line. Each uses FITS files and text configuration files as input and produces one or more FITS files and log files as output. These modules are strung together with a single PERL script. The actual calibration data needed to reduce a given data frame is produced via "calibration pipelines." A raw IRAC image is thus "passed" between successive modules, and at each step becomes closer and closer to a finished, fully reduced BCD image. A full description of the pipeline modules is available on the Spitzer website ${ }^{9}$. To mitigate commonly found artifacts of straylight, saturation, and column pulldown, an artifact correction pipeline was created and acts on the reduced $\mathrm{BCD}$ to created a "Corrected $\mathrm{BCD}$ " or $\mathrm{CBCD}$ image. The artifact correction is good, but imperfect due to being derived from the $\mathrm{BCD}$ in question. Both the $\mathrm{BCD}$ and $\mathrm{CBCD}$ files are delivered to the user as science quality data.

Warm mission IRAC data are processed using the same pipeline and methodology as the cold IRAC data. The changes in temperature and bias levels described above affected artifacts in the IRAC images, and therefore the majority of modifications to the pipeline come in the artifact correction pipeline discussed below in greater detail.

\subsection{Pipeline Changes}

At the temperatures used in the cryogenic mission, the InSb arrays used for the $3.6 \mu \mathrm{m}$ and $4.5 \mu \mathrm{m}$ channels suffered from an effect known as "muxbleed". This is believed to be a result of operating the arrays at these unusually cold temperatures of $15 \mathrm{~K}$. When a bright source is read out, the cold electronics do not return to their quiescent state for a considerable length of time. The result is a ghosting along the pixel readout channels, sometimes referred to as "ant trails" (Figure 4). The effect is easily noticeable against a low background (such as a dark bias measurement), and can extend the full length of the array. The muxbleed flux is not real - it is not "borrowed" from the actual source and as such needs to be accounted for, or removed, unlike CTE smearing in CCDs. This artifact was detected and corrected within the original cryogenic pipeline. With the arrays at a higher temperature during the warm era, the muxbleed artifact was no longer observed and therefore the correction was turned off in the pipeline.

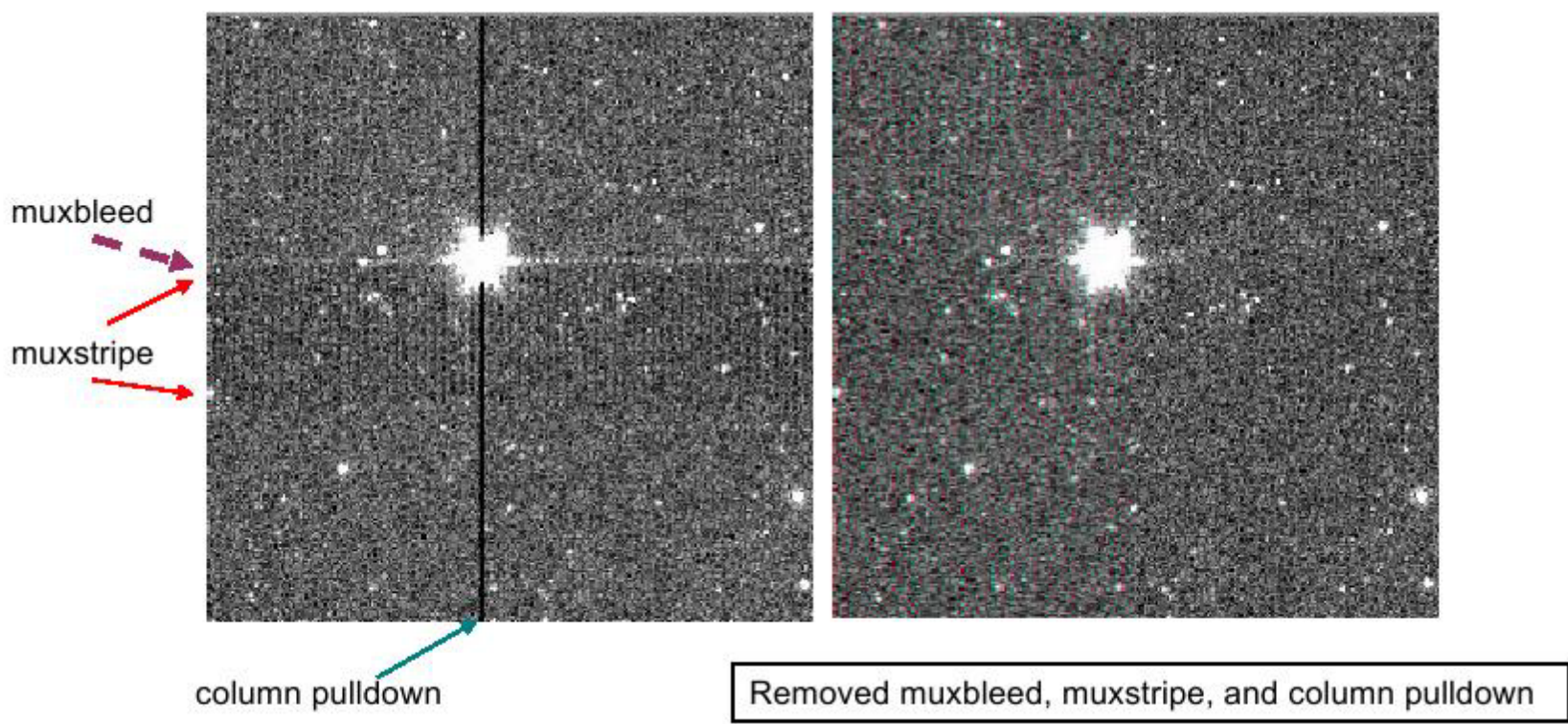

Figure 4. Removal of artifacts within a $4.5 \mu \mathrm{m}$ cryogenic image. Muxbleed and muxstripe are no longer needed in the warm data reduction pipeline. Column Pulldown remains (Section 3.2). 


\subsection{Column Pulldown}

When a bright star or cosmic ray on the array reaches a level above approximately $30,000 \mathrm{DN}$, there is a change in the intensity of the column in which the signal is found. In channels 1 and 2, the intensity is reduced throughout the column (thus the term "column pull-down"); see Figure 4. When the effect occurs, it shifts the intensities of the pixels above and below the position of the "guilty" source, within the same column. In the cryogenic mission, column pulldown was well characterized by a DC offset (different above and below the source). This artifact was well corrected as part of the enhanced IRAC pipeline. Using the "truth image" of the sky background for each column, a robust weighted DC offset was determined. This is a simple offset between the affected column and the estimated background value. This offset is determined separately above and below the triggering source. The offset was then applied to the affected column and saved into the CBCD image, thereby removing the bias shift from all the pixels in the column.

In the warm mission, column pulldown is triggered by slightly fainter sources $(20,000-30,000 \mathrm{DN})$ and has a more complex behavior. The detection method was changed in the artifact pipeline. In the cryogenic mission, potential triggering pixels were located based a fluence threshold, whereas in the warm mission affected columns are identified by looking for columns that are statistically fainter than their neighbors. The new warm mission method is more effective in identifying the artifacts because the triggering threshold appeared to vary significantly with frametime and background level in testing. However, this neighboring-columns method is more easily fooled by structure in the background. As shown in Figure 5, the pulldown is well modeled by separate exponential fits above and below the source. The fit is determined iteratively from the difference of the artifact-corrupted image and an estimate of the true sky derived using a Gaussian interpolate. This adjustment in the fit was tested and added to the pipeline.
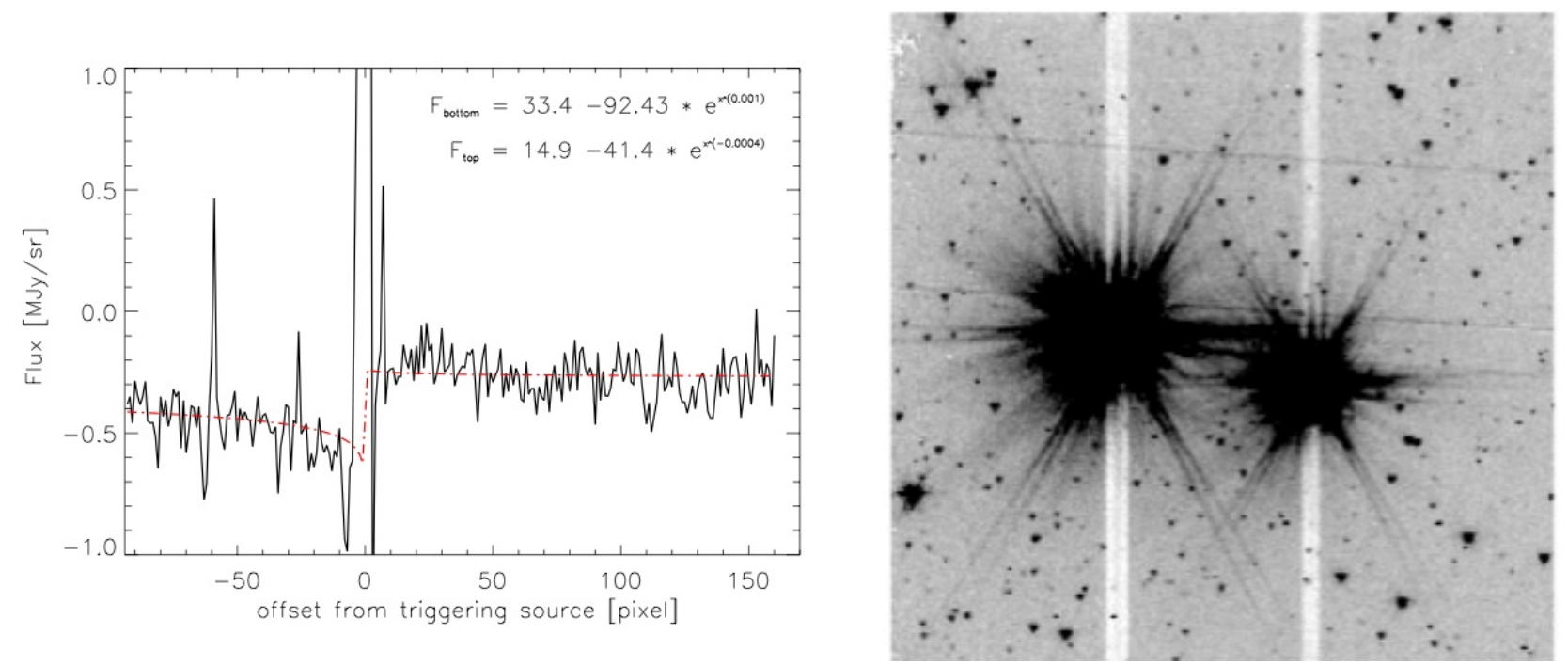

Figure 5. Example of a fit (dotted line) to column pulldown from warm mission data (left). The column pulldown in the image (right) is triggered by a $5^{\text {th }}$ and $7^{\text {th }}$ magnitude star, respectively. The fitted pulldown (left) is the one triggered by the $7^{\text {th }}$ magnitude star.

\subsection{Latent or Persistent Images}

The terms "persistent image", "residual image", and "latent image" are used interchangeably to describe the contamination of an IRAC image by a bright source from a previous exposure. When a pixel is illuminated, a small fraction of the photoelectrons become trapped. The traps have characteristic decay rates, and can release a hole or electron that accumulates on the integrating node long after the illumination has ceased. The warm mission short-term residual images are different in character than the cryogenic residuals, as the behavior of the trap populations is a function of the impurity type and array temperature. During the cryogenic mission, in all arrays, the longest e-folding decay time is about $1000 \mathrm{sec}$ for the short-term traps. For the warm mission, residuals are $<0.01 \%$ of the fluence of the illuminating source after 60 seconds. 


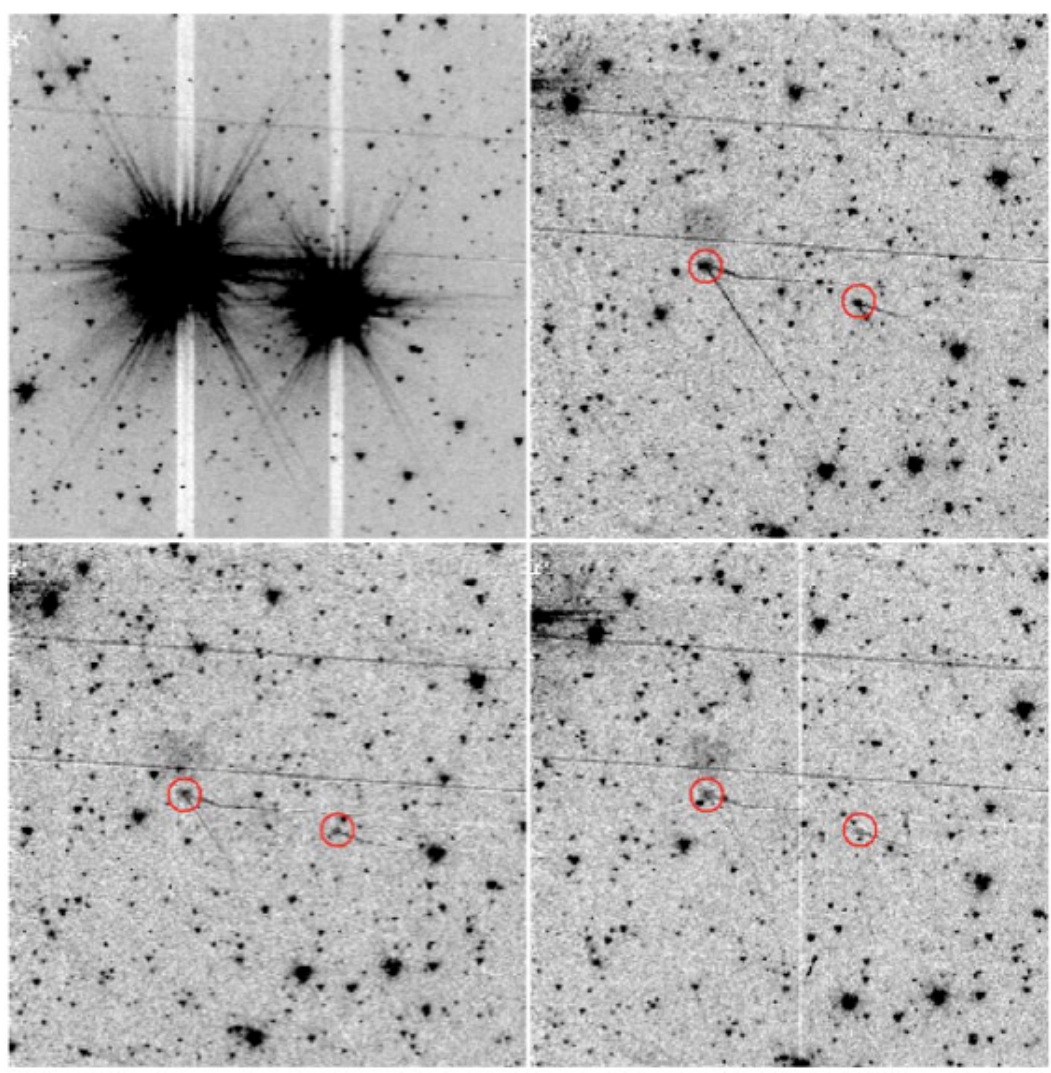

Figure 6. $3.6 \mu \mathrm{m}, 12$ second frames spaced about 30 seconds apart starting with an image of $5^{\text {th }}$ and $7^{\text {th }}$ magnitude stars (upper left), then subsequent dithers (upper right, lower left, lower right) showing the decay of residual images from those intermediate brightness stars. Also visible are slew latents (streaks across image above the stellar residuals) from brighter sources that happened to cross the array while slewing to the targets. Smaller and decaying slew latents are visible from the $5^{\text {th }}$ and $7^{\text {th }}$ magnitude stars

Channel 1 and channel 2 have different persistent image responses in the warm mission data. There are no long-term residual images that last for weeks, such as those seen in channel 1 data during the cryogenic mission. These long term latents in the cryogenic mission were usually removed by annealing the arrays. The arrays are not annealed during the warm mission as there is no evidence that annealing removes residual images (the arrays currently operate at nearly the old annealing temperature), and all residual images decay in a reasonably short time scale compared to those mitigated by annealing in the cryogenic mission.

Table 1: Warm mission measure of latent duration. The length of time a persistence image lasted $3 \sigma$ above the noise.

\begin{tabular}{|c|c|c|}
\hline $\begin{array}{l}\text { Star } \\
\text { magnitude }\end{array}$ & $\begin{array}{l}\text { Channel 1 residual duration } \\
\text { (hours) }\end{array}$ & $\begin{array}{l}\text { Channel 2 residual duration } \\
\text { (hours) }\end{array}$ \\
\hline 1 & 10 & 0.1 \\
\hline 2 & 7 & $<0.1$ \\
\hline 3 & 3.5 & $<0.1$ \\
\hline 4 & 1.5 & $<0.1$ \\
\hline
\end{tabular}

Table 1 gives a rough idea of warm mission latent durations for the brightest stars. Durations should not be taken as exact because they also depend on the background levels in the images that will change from one AOR to the next. This example comes from several bright star observations during testing and starts with $12 \mathrm{~s}$ observations. 
Channel 1 residual images last for minutes to hours, depending on the brightness of the original source and the background levels in the subsequent images. Figure 7 shows this persistent image behavior for a strongly saturated first magnitude star. The residual image decay is exponential in character, as expected for trapped electron decay rates. The decay rate is constant for all sources, so that while residual images from brighter sources take longer to decay below the background level, all the persistent images decay at the same rate. These rates have been implemented for residual image flagging in the warm mission IRAC pipeline.

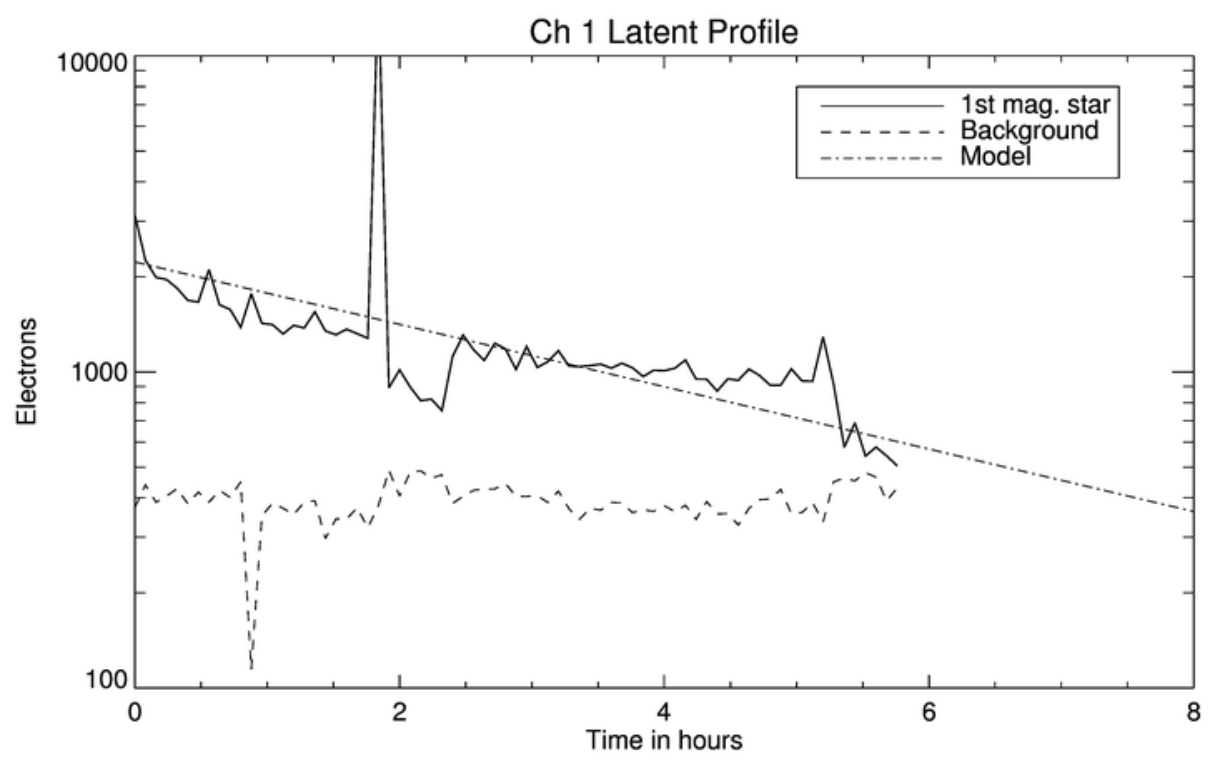

Figure 7: Residual image brightness decay as a function of time interval since exposure to a first magnitude source at $3.6 \mu$ m. The residual is compared to three times the noise in the sky background as measured in an equivalent aperture. The fitted exponential decay function is plotted as the dot-dashed line. These curves have been smoothed to mitigate flux jumps due to sources at the position of the original source in subsequent images.

A consequence of the intermediate-term (hours) residual images is that it is possible for observations from a previous AOR to produce residual images. This is a paradigm shift in the pipeline methodology that originally required each BCD to be processed independently. Now the residual image flagging module correspondingly tracks residuals from one AOR to the next. Given the original brightness of the saturation-corrected source, and the decay time calculated with the exponential decay rate, the pipeline flags all residual images until their aperture fluxes in subsequent frames are less than three times the background noise in each image. Each image in each AOR observed is checked for residual images from all previous observations within the observing campaign. These are flagged in the mask files associated with each $\mathrm{BCD}$, and therefore the masks can be used to discriminate against possible residuals when searching for faint astrophysical objects.

Channel 2 residual images decay much faster than those in channel 1 and last only a few minutes $(<10)$ for even the brightest stars. Therefore, the pipeline flagging for channel 2 does not cross AORs. Channel 2 residuals start out as positive, but then become negative. The timing of the switch from positive to negative depends on the exposure time and brightness of the source.

For extremely bright sources, residuals are produced even when the source is not imaged on the array. Residuals at 3.6 and 4.5 microns can be produced during slews from one science target to another and from one dither position to the next. These slew residuals appear as linear features streaking across IRAC images. Note that the pipeline cannot flag these slew residuals, as there is no reasonable way of tracking the appearance of bright sources relative to the moving telescope pointing. This is another unfortunate consequence of shutterless operation for IRAC.

Observations contaminated by residual images can often be corrected with the data themselves. If the observations were well dithered, it is likely that the persistent image artifacts will be rejected as outliers when building the mosaic. The pipeline produces a median stack of all images in an observation request that can be downloaded from the Spitzer 
Heritage Archive, and the median can often be used to identify pixels that are affected by residual images. Residual images can often be at least partially mitigated by subtracting the normalized median stack image (made with object and outlier rejection).

\section{SUMMARY}

The warm IRAC mission is producing science data of comparable quality to the cryogenic mission for the 3.6 and $4.5 \mu \mathrm{m}$ channels and can continue to make profound scientific discoveries for many years. The basic calibration pipeline and artifact correction pipeline have been adjusted to mitigate well-understood instrumental signatures. Warm data are at worst $10 \%$ less sensitive than cryogenic. The data are currently absolutely calibrated to $4 \%$. Warm IRAC has demonstrated the sensitivity necessary to detect galaxies at high redshift and the photometric stability to make high precision relative photometry observations of transits of approximately Earth sized planets around $\mathrm{M}$ stars and characterize the thermal phase curves of larger exoplanets.

The authors acknowledge the efforts of our colleagues in the IRAC instrument and instrument support teams in maintaining and characterizing the IRAC instrument, as well as the operations teams at the Spitzer Science Center, JPL and Lockheed Martin. This work is based on observations made with the Spitzer Space Telescope, which is operated by the Jet Propulsion Laboratory, California Institute of Technology under a contract with NASA. Support for this work was provided by NASA through an award issued by JPL/Caltech.

\section{REFERENCES}

[1] Werner, M. N., Roellig, T. L., Low, F. J., Rieke, G. H., Rieke, M., Hoffmann, W. F., Young, E., et al., “The Spitzer Space Telescope Mission", ApJS, 154, 1-9 (2004).

[2] Fazio, G. G., Hora, J. L., Allen, L. E., Ashby, M. L. N., Barmby, P., Deutsch, L. K., Huang, J.-S., et al., "The Infrared Array Camera (IRAC) for the Spitzer Space Telescope”, ApJS, 154, 10-17 (2004).

[3] Hora, J. L., Fazio, G. G., Allen, L. E., Ashby, M. L. N., Barmby, P., Deutsch, L. K., Huang, J.-S., et al., "In-flight performance and calibration of the Infrared Array Camera (IRAC) for the Spitzer Space Telescope", Proc. SPIE 5487, 77-92 (2004).

[4] Houck, J. R., Roellig, T. L., van Cleve, J., Forrest, W. J., Herter, T., Lawrence, C. R., Matthews, K., Reitsema, H. J., et al., "The Infrared Spectrograph (IRS) on the Spitzer Space Telescope", ApJS, 154, 18-24 (2004).

[5] Rieke, G. H., Young, E. T., Engelbracht, C. W., Kelly, D. M., Low, F. J., Haller, E. E., Beeman, J. W., et al., "The Multiband Imaging Photometer for Spitzer (MIPS)", ApJS, 154, 25-29 (2004).

[6] McMurtry, C. W., Pipher, J. L., Forrest, W. J., "Spitzer space telescope: dark current and total noise prediction for $\mathrm{InSb}$ detector arrays in the infrared array camera (IRAC) for the post-cryogen era”, Proc. SPIE 6265, 08 (2006).

[7] Storrie-Lombardi, L. J., et al., "The Science Opportunities of the Warm Spitzer Mission”, AIP, 943 (2007).

[8] Carey, S., Surace, J., Lacy, M., Glaccum, W., Lowrance, P., Hora, J. L., and Willner, S. "Stability of the Infrared Array Camera for the Spitzer Space Telescope", Proc. SPIE 7010, 70102V-70102V-8 (2008).

[9] IRAC Instrument Support Teams, "IRAC Instrument Handbook," March 2012, http://irsa.ipac.caltech.edu/data/SPITZER/docs/irac/iracinstrumenthandbook

[10] Ingalls, J.,Krick, J., Carey, S., Laine, S., Surace, J., Glaccum, W., Grillmair, C., Lowrance, P., "Intra-pixel gain variations and high precision photometry with the InfraRed Array Camera (IRAC)", this proceedings 